Prävention in der Hausarztpraxis

\section{Wie motivieren Sie Patienten zu einem gesünderen Leben?}

In einer Gesellschaft, die immer älter und immer dicker wird, muss sich die Medizin mehr der Primärprävention und der Gesundheitsförderung verschreiben. Mit welchen Maßnahmen der Hausarzt dazu beitragen kann, erklärt der Vorsitzende der Deutschen Akademie für Präventivmedizin e.V. (DAPM), Dr. med. Johannes Scholl aus Rüdesheim am Rhein.

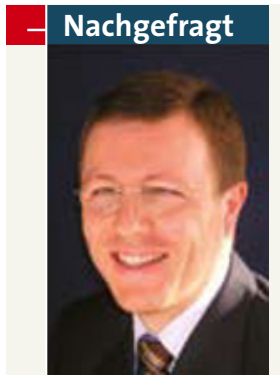

bei Dr. med. J. Scholl

„Allein die Ermahnung des Hausarztes bewegt zusätzliche $3 \%$ der Raucher zum Nikotinstopp."

MMW: Was lässt sich mit Prävention erreichen?

Scholl: Viele Herzinfarkte und Diabeteserkrankungen wären vermeidbar, wenn man die bekannten modifizierbaren Risikofaktoren angehen würde. Etwa 80-90\% des kardiovaskulären Risikos hätten wir dadurch in der Hand. Anfang des Jahres wurde eine deutsche Studie veröffentlicht, in der 80\% der Typ-2-Diabetiker innerhalb von drei Wochen ohne eine Verschlechterung ihrer Stoffwechsellage ihre oralen Antidiabetika absetzen konnten - einfach indem sie ihre Ernährung umstellten und täglich körperlich aktiv waren.

MMW: Wie kann der Hausarzt bei seinen Patienten Prävention unterstützen? Scholl: Prävention wird in Deutschland vorwiegend unter dem medikamentösen Aspekt gesehen, aber das ist nur die halbe Wahrheit. Zunächst müssen wir am Lebensstil ansetzen, bei der Bewegung und bei der Ernährung. Die DAPM grenzt sich bewusst von Konzepten ab, die mit unbewiesenen Versprechungen Nahrungsergänzungsmittel oder Diätprodukte zur Prävention verkaufen wollen. Wer als Hausarzt Prävention vermitteln will, sollte beispielsweise wissen, wie er einen ein- fachen Trainingsplan für seine Patienten erstellt und welches der aktuelle Stand in der Ernährungstherapie ist. Außerdem gehört auch der Umgang mit Suchtverhalten dazu, z. B. Nikotinentwöhnung.

MMW: Eine große Schwierigkeit besteht darin, die Patienten zur Änderung ihrer Lebensgewohnheiten zu motivieren. Wie kann das gelingen?

Scholl: Sehr wichtig ist, die offensichtlichen Probleme der Patienten überhaupt anzusprechen. Damit schafft man bei den Patienten Bewusstsein dafür, dass sie etwas für sich selbst tun können. Allein die Ermahnung des Hausarztes „Das Rauchen schadet Ihnen, Sie sollten damit aufhören " bewegt schon zusätzliche 3\% der Raucher zum Nikotinstopp. Wenn die Patienten darüber hinaus verständliche Erläuterungen über wissenschaftliche Zusammenhänge bekommen, haben sie oft ein Aha-Erlebnis und sind besser zu motivieren.

MMW: Welche Patienten sollte der Hausarzt auf die Möglichkeiten zur Prävention ansprechen?

Scholl: Alle Patienten mit familiärer Belastung für Herz-Kreislauf-Erkrankungen, Diabetes oder Kolonkarzinom, aber auch Frauen, die während der Schwangerschaft Diabetes hatten, sowie übergewichtige bzw. adipöse Patienten sollten über geeignete Präventionsmaßnahmen informiert werden. Generell sollte jeder Mensch spätestens mit 30 Jahren wissen, ob und welche Risikofaktoren bei ihm vorliegen, weil man dann noch rechtzeitig gegensteuern kann.

MMW: Prävention ist im deutschen Gesundheitswesen kaum vorgesehen. Kann

\section{Zertifizierter Kurs}

Fortbildung in Präventivmedizin

Die Deutsche Akademie für Präventivmedizin e.V. bietet vom 23.9.1.10.2006 einen 80-stündigen, von der LÄK Hessen mit 8o Punkten zertifizierten Fortbildungskurs an, der praxisrelevant und nach evidenzbasierten Kriterien das aktuelle Wissen über Prävention vermittelt. Weitere Kurse sind für 2007 geplant.

Weitere Informationen: Deutsche Akademie für Präventivmedizin e. V., Eibinger Str. 9, 65385 Rüdesheim, Tel.: 06722/710980o, www.akaprev.de.

sie sich für den niedergelassenen Arzt überhaupt lohnen?

Scholl: Die gesetzlichen Krankenkassen erstatten nur einen sehr kleinen Leistungsumfang, z. B. die Check-up-Untersuchungen ab dem 35. Lebensjahr. Die Krankenversicherung ist in der Regel nur leistungspflichtig, wenn der Patient Beschwerden oder Krankheiten hat. Heute müssen wir aber im Sinne der Eigenverantwortung weiterdenken. Ich sehe die Bewertung von Risikofaktoren oder die Ernährungs- und Trainingsberatung als sinnvolle Präventionsdienstleistungen an, mit denen der Arzt als seriöse IGeL-Leistung ein angemessenes Honorar erwirtschaften kann.

- Interview: Dr. Judith Neumaier 